\title{
FATIGUE CRACK GROWTH FROM A CIRCULAR NOTCH UNDER HIGH LEVELS OF BIAXIAL STRESS
}

\author{
Hong Youshi ${ }^{1}$, M. W. BRown ${ }^{2}$ and K. J. MilleR ${ }^{2}$ \\ ${ }^{1}$ Institute of Mechanics, Chinese Academy of Sciences, Beijing 100080, People's Republic of China and \\ ${ }^{2}$ Department of Mechanical and Process Engineering. The University of Sheffield, Mappin Street, \\ Sheffield S1 3JD, England
}

(Received in final form 10 February 1992)

\begin{abstract}
A series of experiments have been conducted on cruciform specimens to investigate fatigue crack growth from circular notches under high levels of biaxial stress. Two stress levels $\left(\Delta \sigma_{1}=380\right.$ and $560 \mathrm{MPa})$ and five stress biaxialities $\left(\lambda=+1.0,+0.5,0,-0.5\right.$ and -1.0 ; where $\left.\lambda=\sigma_{2} / \sigma_{1}\right)$ were adopted in the fatigue tests in type 316 stainless steel having a monotonic yield strength of $243 \mathrm{MPa}$. The results reveal that fatigue crack growth rates are markedly influenced by both the stress amplitude and the stress biaxiality. A modified model has been developed to describe fatigue crack growth under high levels of biaxial stress.
\end{abstract}

\section{INTRODUCTION}

Many fatigue failures of engineering structures and components have been associated with the multiaxial loading histories that they experienced. Of these examples, it is frequently found that fatigue cracks had initiated from notches, due to the effects of stress concentration. Therefore, fatigue crack growth from notches under biaxial or triaxial stress states has attracted increasing academic attention in recent years.

To describe the propagation rate of fatigue cracks and predict fatigue life, the well-known Paris law [1] has achieved great success in describing long cracks, i.e. cracked bodies experiencing low levels of stress due to cycling in the elastic loading regime, where linear elastic fracture mechanics (LEFM) can apply. However, when a crack initiates and then propagates from a notch root under high cyclic loading conditions, it is called a short crack because of the significant amount of plasticity involved. This is the case for low-cycle fatigue, where the stress and the strain range experienced are beyond the small-scale yielding conditions required by LEFM, and the original Paris law is no longer effective. It is therefore inevitable that elastic-plastic fracture mechanics (EPFM) must be introduced and elastic-plastic parameters must be taken into acccount when modelling fatigue crack propagation under high levels of cyclic stress. Such parameters include (a) $\Delta K_{\epsilon}$, the strain intensity factor [2,3], (b) $\Delta J$, the cyclic $J$ integral [4,5], (c) $\delta$, the crack tip opening displacement [6] and (d) $r_{p}$, the plastic zone size [7,8]. So a variety of EPFM approaches to the low-cycle fatigue problem have been developed, using the above parameters to describe crack growth rate under uniaxial cyclic loading [2-8].

The behaviour of fatigue cracks propagating from notches under low levels of biaxial stress has been reported previously $[9,10]$, showing that although both the stress biaxiality and the stress amplitude affect fatigue response, the crack growth rates still obey the Paris law. But in the regime of high biaxial stress and strain, the interaction of fatigue cracks with notches still remains an area of argument and research. A better understanding of the physical phenomena involved and the availability of models for evaluating fatigue crack growth in this regime are, therefore, of great importance. 
The present study has been carried out to develop an expression describing the growth rate of fatigue cracks from notches under high levels of biaxial stress, based on an experimental investigation using cruciform specimens with central circular notches which are subjected to loading at different biaxialities. Fatigue crack lengths were measured by both the potential drop technique and a travelling microscope. The deformation, i.e. the displacement on both loading axes, was measured by a pair of extensometers clamped to one side of the specimen. The biaxial cyclic stress-strain relationship was also recorded from the same type of specimen without a notch. The cyclic strain hardening exponents of the $\Delta \sigma_{1}$ versus $\Delta \epsilon_{\mathrm{lp}}$ relationships obtained were used to develop an EPFM model, and the experimental results are compared with crack growth rate predictions developed from the model.

\section{A MODIFIED MODEL}

About two decades ago, an original model for fatigue crack propagation under high levels of cyclic stress was proposed by Tomkins [7], based on the concept that an increment of fatigue crack surface is formed by shear de-cohesion in each load cycle, and that crack growth rate, $\mathrm{d} a / \mathrm{d} N$, is therefore proportional to plastic zone size, $r_{\mathrm{p}}$, and to the range of cyclic plastic strain, $\Delta \epsilon_{\mathrm{lp}}$, in the direction normal to the fatigue crack surface, i.e.

$$
\frac{\mathrm{d} a}{\mathrm{~d} N}=\int_{0}^{\Delta c_{1 \mathrm{p}}} r_{\mathrm{p}} \mathrm{d} \epsilon_{1 \mathrm{p}}
$$

Using Dugdale's solution [11] for $r_{\mathrm{p}}$ for a Mode I crack of length $2 a$ in an infinite plate with remote applied stress normal to the crack plane, $\sigma_{1}$,

$$
r_{\mathrm{p}}=a\left[\sec \left(\frac{\pi \sigma_{1}}{2 \sigma_{0}}\right)-1\right]
$$

In the fatigue crack propagtion situation, Tomkins chose to use the ultimate tensile strength of the material, $\sigma_{\mathrm{u}}$, as the cohesive stress, $\sigma_{0}$, which represents the flow stress of cyclically strain hardened material at the crack tip. When $\sigma_{1} / \sigma_{\mathfrak{u}}<0.5$, using the first term of the series expansion of equation (2), equation (1) can be expressed, for a cyclic cohesive stress $2 \sigma_{u}$, as (also see [12])

$$
\frac{\mathrm{d} a}{\mathrm{~d} N}=\frac{\pi^{2}}{8(1+2 n)}\left(\frac{\Delta \sigma_{1}}{2 \sigma_{\mathrm{u}}}\right)^{2} \Delta \epsilon_{\mathrm{lp}} a
$$

where $\Delta \sigma_{1}$ and $\Delta \epsilon_{1 \mathrm{p}}$ obey a power law formulation under uniaxial loading,

$$
\Delta \epsilon_{1}=\Delta \epsilon_{1 \mathrm{e}}+\Delta \epsilon_{\mathrm{lp}}=\frac{\Delta \sigma_{1}}{E}+\left(\frac{\Delta \sigma_{1}}{k}\right)^{1 / n}
$$

Note that equation (3) has been derived such that it is only applicable to a Mode I crack in a large plate.

In order to extend the model to be applicable to the case of fatigue crack advance from notch roots, we adopt an approximate analytic solution of plastic zone size for cracks emanating from a circular notch [13]

where

$$
\frac{\sigma_{1}}{\sigma_{0}}=\frac{2}{\pi} \cdot \frac{1}{1+F} \arccos \left(\frac{a}{a+r_{\mathrm{p}}}\right)
$$

$$
F=\frac{m^{2}}{2(1-m)\left(1+m^{2}\right)^{2}}\left[\left(3+3 m-m^{2}+3 m^{3}\right)-\lambda\left(3-5 m+3 m^{2}-m^{3}\right)\right]
$$




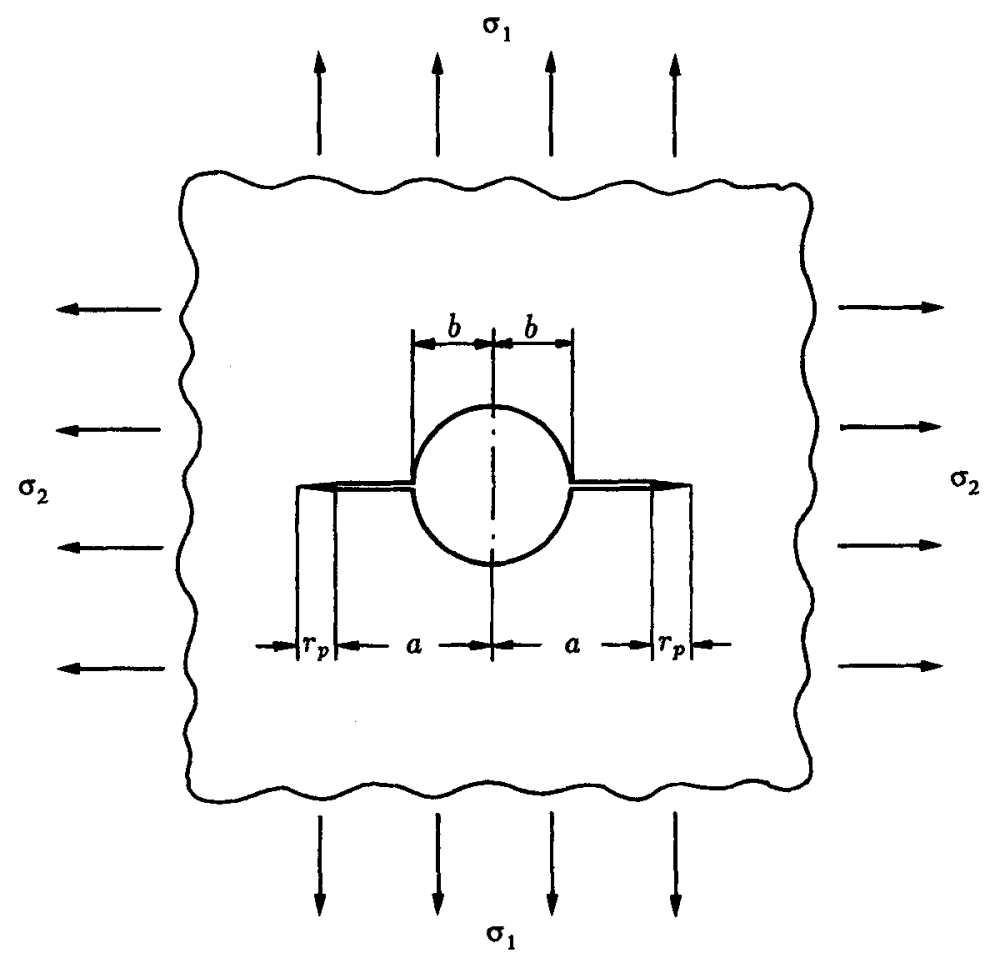

Fig. 1. Schematic showing the geometry of cracks emanating from a circular notch.

in which $\lambda$ is a measure of stress biaxiality $\left(\lambda=\sigma_{2} / \sigma_{1}\right.$, where $\sigma_{2}$ is the stress parallel to the crack surface for a plane stress condition) and $m$ is a parameter depending upon notch size and crack length, given by

$$
m=\frac{a}{b}-\sqrt{1+\left(\frac{a}{b}\right)^{2}}
$$

with $a, b$ and $r_{\mathrm{p}}$ being defined in Fig. 1. Note that, when calculating plastic zone size $r_{\mathrm{p}}, a$ in equation (7) should be replaced by $a+r_{\mathrm{p}}$ in obtaining the corresponding value of $m$. As in the Tomkins' model, $\sigma_{u}$ is regarded as $\sigma_{0}$ in this case. For $\sigma_{1} / \sigma_{u}<0.5$, equation (5) can be written as

$$
r_{\mathrm{p}} \doteq a \frac{\pi^{2}}{8}\left(\frac{\sigma_{1}}{\sigma_{\mathrm{u}}}\right)^{2}(1+F)^{2} .
$$

Substituting equation (8) into equation (1), we therefore have

$$
\frac{\mathrm{d} a}{\mathrm{~d} N}=\frac{\pi^{2}}{8} \cdot \frac{(1+F)^{2}}{(1+2 n)}\left(\frac{\Delta \sigma_{1}}{2 \sigma_{\mathrm{u}}}\right)^{2} \Delta \epsilon_{1 \mathrm{p}} a .
$$

This is a modification of Tomkins' formula allowing for fatigue crack extension from a circular notch under biaxial loading.

Equations (9) and (3) provide a reasonable crack propagation law in the low-cycle fatigue regime, but some open questions remain. The first important argument is the definition of $\Delta \epsilon_{1 p}$, a key factor in the model. In Tomkins' development, the cyclic plastic strain far from a crack was considered. However, fatigue crack extension is closely related to the deformation generated inside the plastic 
zone of a cracked notch, which must be taken into account in the modelling. This will be further discussed later in the paper.

\section{BIAXIAL FATIGUE TESTING METHOD}

The cruciform specimens (Fig. 2) used in this investigation were manufactured from solution annealed AISI 316 austenitic stainless steel with the chemical composition of (wt \%) C 0.049 , Mn 1.36, P 0.023, S 0.018, Si 0.54, Cr 17.26, Ni 11.20, Mo 2.15 and remainder Fe. The tensile properties of the material at room temperature were $0.2 \%$ proof stress $243 \mathrm{MPa}$, ultimate tensile strength $597 \mathrm{MPa}$, elongation $68 \%$ and reduction in area $71 \%$.

Fatigue tests were carried out on a Mayes servohydraulic biaxial test facility. The machine is capable of producing two alternating loads, via two pairs of servohydraulic actuators acting on orthogonal axes of a cruciform specimen. The cyclic loads in each axis are controlled independently, so that different stress biaxialities may be exerted on the working section of the cruciform specimen. A pair of very shallow cuts were introduced by spark erosion (typically $0.2 \mathrm{~mm}$ deep), located on opposite sides of the central notch of $16 \mathrm{~mm}$ diameter, encouraging Mode I cracks to propagate preferentially along a horizontal line, i.e. the $x$-axis. The circular notch was produced by spark erosion to avoid the introduction of residual stresses.

Two stress ranges were applied in the tests: (a) $\Delta \sigma_{1}=380 \mathrm{MPa}$ and (b) $\Delta \sigma_{1}=560 \mathrm{MPa}$. All tests were conducted at a stress ratio $R=\sigma_{\min } / \sigma_{\max }=-1$ and a frequency between 0.1 and $0.2 \mathrm{~Hz}$. The biaxial stresses were kept in constant proportion during the loading cycle. For the first stress level,
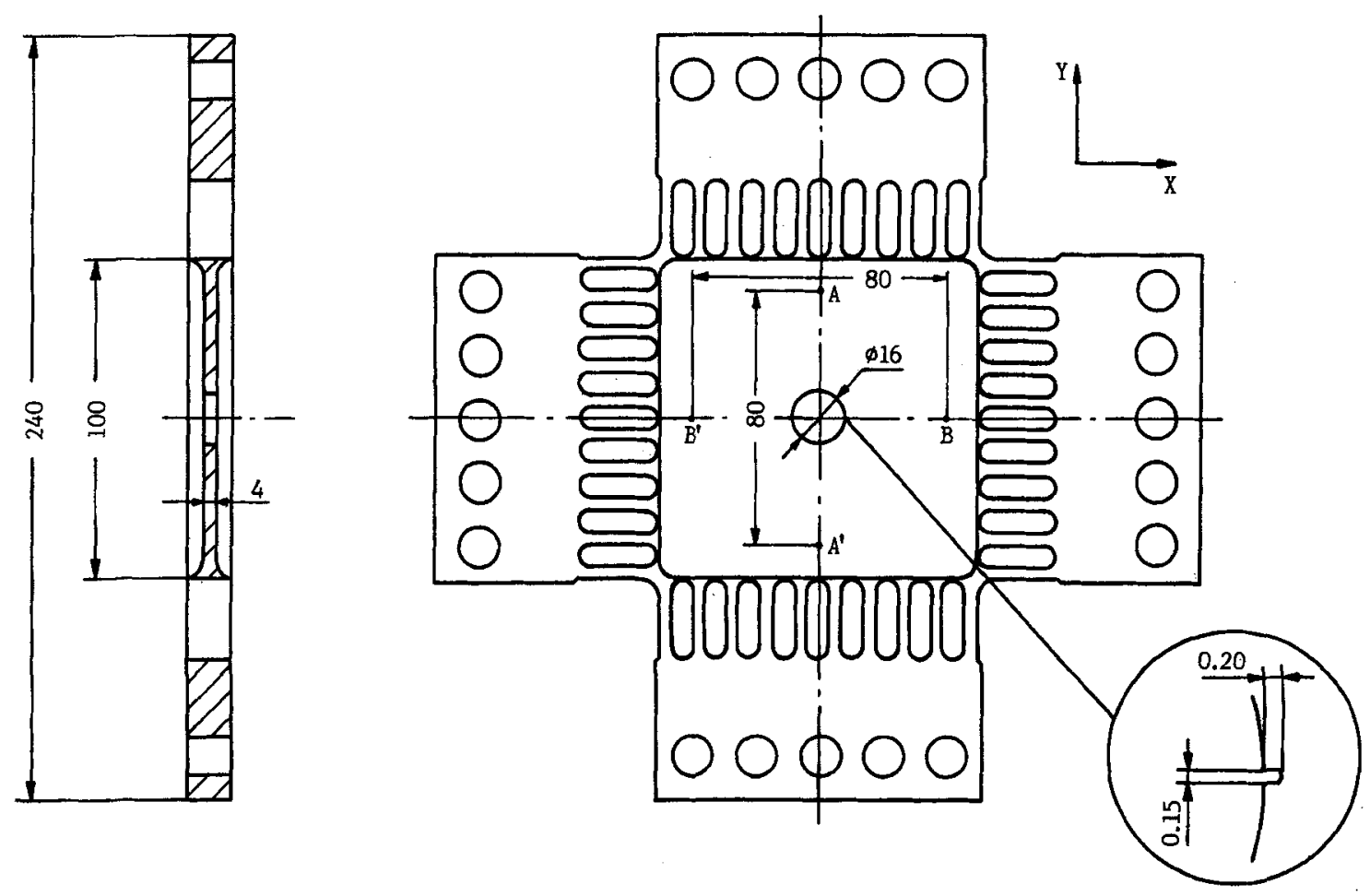

Fig. 2. Cruciform specimen geometry (dimensions in $\mathrm{mm}$ ). 
five stress biaxialities were adopted: $\lambda=+1.0,+0.5,0,-0.5$ and -1.0 , whereas for the second stress level, only three biaxialities were chosen: $\lambda=+1.0,+0.5$ and 0 , since the specimen would be broken after very few cycles of loading at the higher stress amplitude at $\lambda=-1.0$ or -0.5 ; the stresses being above or close to the ultimate strength, in the net section of the specimen.

During testing, fatigue crack lengths were monitored by a d.c. potential drop measuring system and a travelling microscope simultaneously. Three pairs of stainless steel leads were spot-welded to the specimen, one pair being far from the central notch ( $35 \mathrm{~mm}$ on each side of the centre line) to provide a reference voltage, and the other two pairs being symmetrically located on the notch perimeter, $4 \mathrm{~mm}$ either side of the centre line. The relatively small distance between the potential leads of each pair resulted in a larger increment of voltage measured between them due to extension of a short crack. This system was able to detect sensitively the initiation of a crack of $0.5 \mathrm{~mm}$. After crack extension beyond this size, the potential ratio increased with crack length steadily and regularly, whereby crack length could be properly monitored. In practice, the potential drop measurements were used to reconcile the microscope readings and interpolate data between some optical observation intervals.

Two extensometers, equipped with specially designed spring supports, were installed on the specimen, held against points $\mathbf{A}$ and $\mathbf{A}^{\prime}$ with respect to the $y$-axis and points $\mathbf{B}$ and $\mathbf{B}^{\prime}$ with respect to the $x$-axis in each case (Fig. 2). Note that the two parallel planes (normal to the $y$-axis) containing $A$ and $A^{\prime}$, respectively, and a second pair of parallel planes (normal to the $x$-axis) containing $B$ and $\mathrm{B}^{\prime}$, respectively, can be regarded as remote boundaries, and the displacements at a given time along different normal lines between each pair of the parallel planes are approximately constant. In this sense, the gauge length changes of both axes were detected by these extensometers and recorded by a data logger. The hysteresis loops of cyclic load versus displacement were also plotted at regular intervals by an $x-y$ recorder.

An additional biaxial cyclic loading test was performed at the corresponding stress biaxialities of $-1,-0.5,0,+0.5$ and +1.0 , using the same geometry of specimen but without a notch, to obtain the cyclic stress-strain relationship of the material. The plastic strains on both axes were easily obtained by the extensometers described above, for a series of increasing stress ranges in steps of $40 \mathrm{MPa}$. The parameters $k$ and $n$ in equation (4) at these five biaxialities were determined by linear regression from the recorded stress and strain data.

\section{EXPERIMENTAL RESULTS}

\section{Cyclic stress-strain relationship}

The measured principal stress and plastic strain ranges $\left(\Delta \sigma_{1}\right.$ versus $\left.\Delta \epsilon_{\mathrm{lp}}\right)$ from the tests to determine the cyclic stress-strain relationship performed at $\lambda=-1.0,-0.5,0,+0.5$ and +1.0 , were analysed for each biaxiality to give the following formulae by linear regression:

$$
\Delta \sigma_{1}=8.95 \times 10^{2} \Delta \epsilon_{\mathrm{lp}}^{0.152}
$$

for $\lambda=-1$ with $260 \leqslant \Delta \sigma_{1} \leqslant 380 \mathrm{MPa}$,

$$
\Delta \sigma_{1}=1.01 \times 10^{3} \Delta \epsilon_{1 \mathrm{p}}^{0.138} .
$$

for $\lambda=-0.5$ with $260 \leqslant \Delta \sigma_{1} \leqslant 380 \mathrm{MPa}$,

$$
\Delta \sigma_{1}=1.89 \times 10^{3} \Delta \epsilon_{1 \mathrm{p}}^{0.181}
$$


for $\lambda=0$ with $380 \leqslant \Delta \sigma_{1} \leqslant 560 \mathrm{MPa}$,

$$
\Delta \sigma_{1}=1.85 \times 10^{3} \Delta \epsilon_{1 \mathrm{p}}^{0.171}
$$

for $\lambda=+0.5$ with $380 \leqslant \Delta \sigma_{1} \leqslant 560 \mathrm{MPa}$, and

$$
\Delta \sigma_{1}=1.98 \times 10^{3} \Delta \epsilon_{1 \mathrm{p}}^{0.180}
$$

for $\lambda=+1.0$ with $380 \leqslant \Delta \sigma_{1} \leqslant 560 \mathrm{MPa}$.

All correlation coefficients for the above equations were larger than 0.990 , indicating that a very good linear tendency exists between $\Delta \sigma_{1}$ and $\Delta \epsilon_{\mathrm{lp}}$ at the five biaxialities.

Fatigue crack growth under biaxial stresses

A presentation of fatigue crack growth under high levels of biaxial stress is given by Fig. 3(a) and (b) for the two stress levels, in terms of crack growth rate plotted against nominal $\Delta K$, where

$$
\Delta K=\Delta \sigma_{1} \sqrt{\pi a}(1+F)
$$

derived in a previous paper [13] for this configuration, the definition of $F$ being given by equations (6) and (7). Note that this expression is applicable to the case of a cracked notch for $(a-b) / b>0.4$ (Fig. 1), whereas for crack extension under this limit, $(1+F)$ was taken from the numerical results of Newman [14] or Xiao et al. [15], for the case of an infinitely wide plate. Obviously, the stress levels used in this study are so high that LEFM premises do not hold, and $\Delta K$ is presented only as a convenient parameter for plotting crack velocity against applied stress amplitude and crack length.
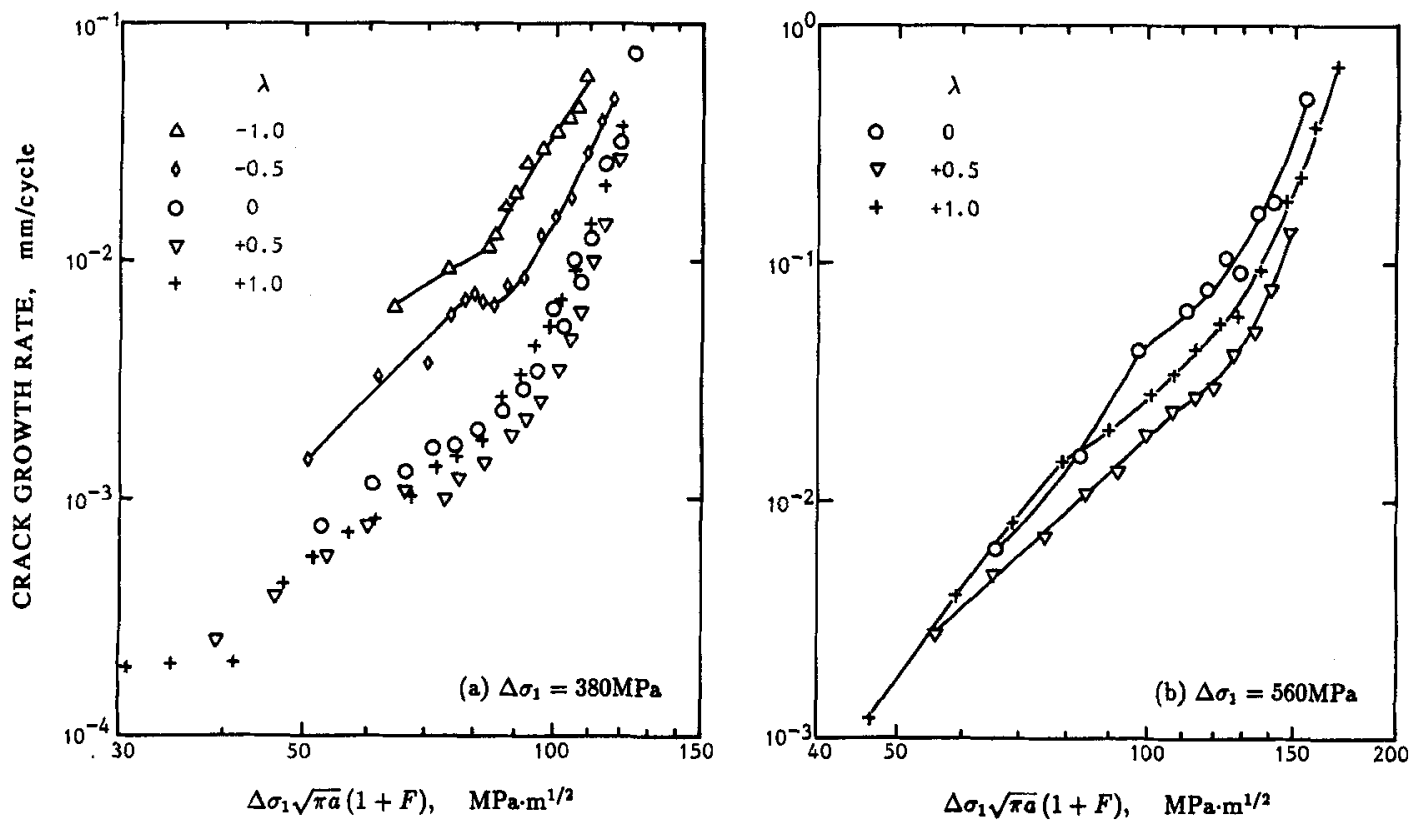

Fig. 3. Fatigue crack growth rate $\mathrm{d} a / \mathrm{d} N$ vs $\Delta \sigma_{1} \sqrt{\pi a}(1+F)$, for (a) $\Delta \sigma_{1}=380 \mathrm{MPa}$ and (b) $\Delta \sigma_{1}=560 \mathrm{MPa}$. 

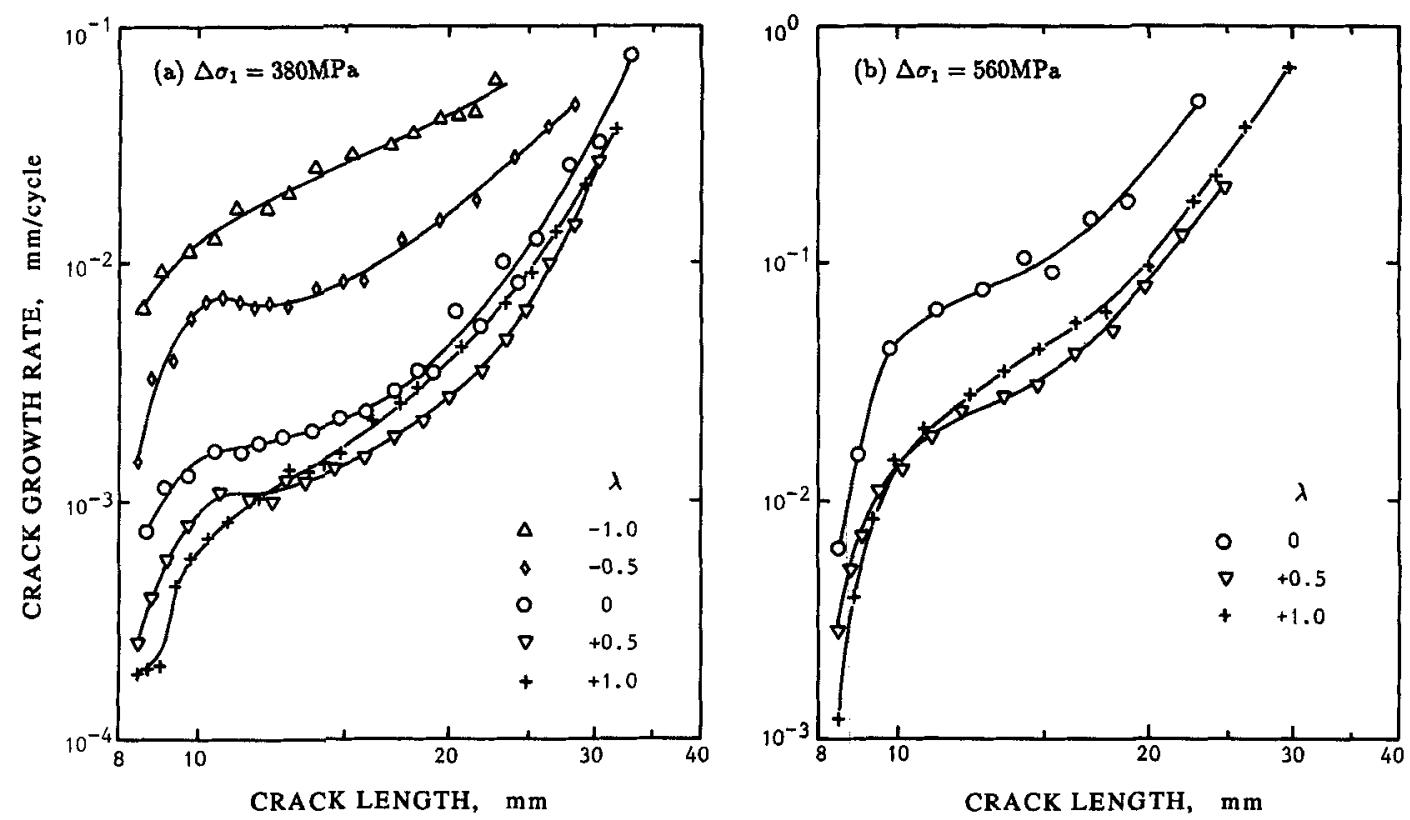

Fig. 4. Fatigue crack growth rate $\mathrm{d} a / \mathrm{d} N$ vs crack length $a$, for (a) $\Delta \sigma_{1}=380 \mathrm{MPa}$ and (b) $\Delta \sigma_{1}=560 \mathrm{MPa}$.

The results are also shown as crack growth rate versus total crack length $a$, as indicated in Fig. 4(a) and (b). Crack growth rates in this investigation were obtained by the secant formula, following ASTM standard E647 [16].

Gauge length displacement during fatigue

Since fatigue cracks for all test specimens propagated close to the $x$-axis, applied strains in the $y$-axis direction are more relevant to Mode I fatigue crack growth. Figure 5(a) and (b) shows the results of displacement measured on the $y$-axis, in relation to crack length, at different stress biaxialities for both stress levels. In fact, the length changes along the central vertical line, as shown in Fig. 5, should be interpreted as the sum of the strains on the $y$-axis plus the crack tip opening displacment (COD).

Figure 6(a) and (b) shows two examples of load-displacement hysteresis loops for $\Delta \sigma_{1}=380 \mathrm{MPa}$ with $\lambda=+1.0$ and $\Delta \sigma_{1}=560 \mathrm{MPa}$ with $\lambda=+0.5$, respectively. It is evident that the minimum point of displacement moves to the right, i.e. towards a greater value, with the increase of loading cycles or crack extension, which is caused mainly by crack closure. This suggests that the maximum point of the displacement is a more representative parameter with regard to fatigue crack propagation, due to the presence of crack closure. Figure 7(a) and (b) shows the variation of $\Delta V_{\mathrm{m}}$ (the net increase of the maximum value of gauge length displacement, $V_{\mathrm{m}}$ ) with crack length for different stress biaxialities at the two stress levels.

\section{DISCUSSION}

Figures 3 and 4 clearly indicate that fatigue crack growth rates under high levels of biaxial stress are affected substantially by both the stress amplitude and biaxiality. Being referred to as nominal 

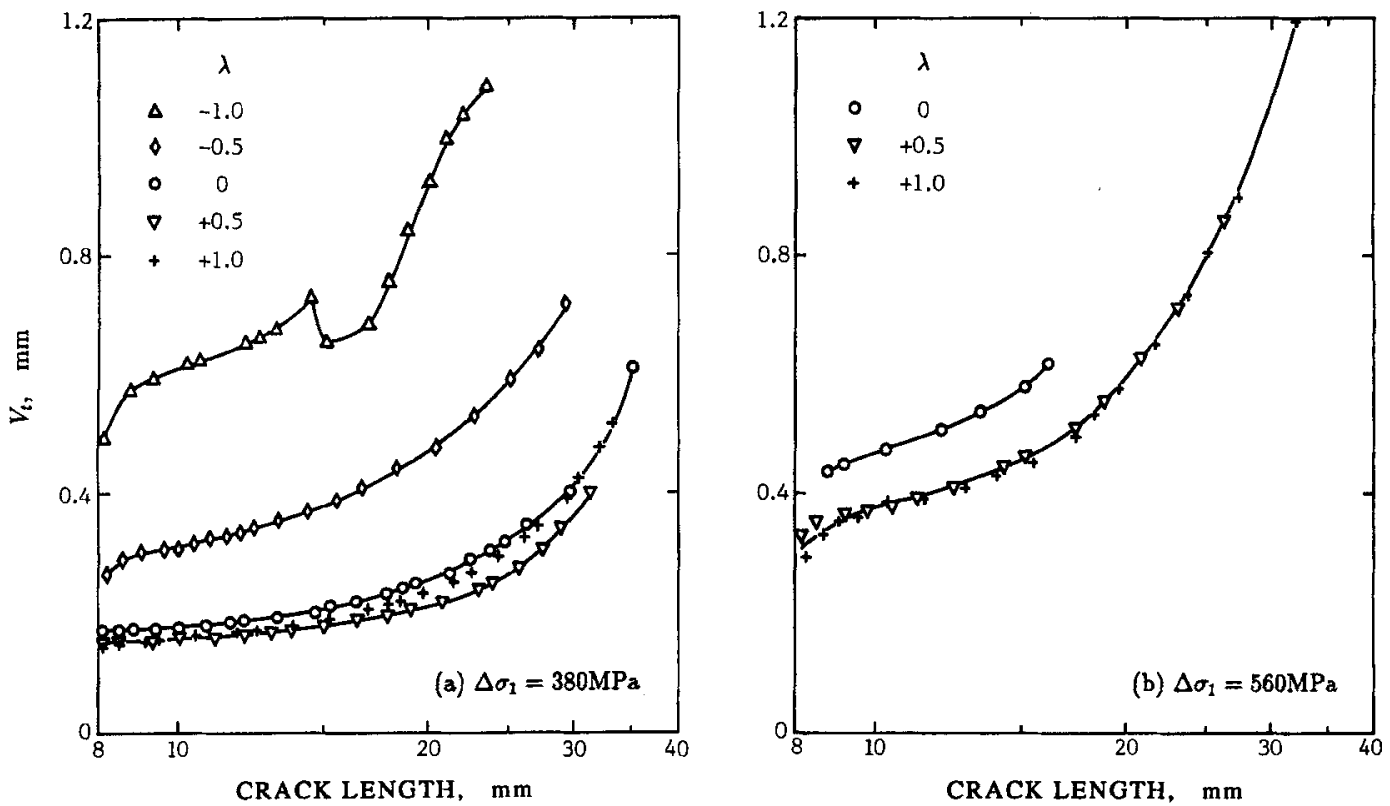

Fig. 5. Total displacement of gauge length for the $y$-axis, $V_{t}$, as a function of crack length $a$, for (a) $\Delta \sigma_{1}=380 \mathrm{MPa}$ and (b) $\Delta \sigma_{1}=560 \mathrm{MPa}$.

$\Delta K$ or crack length $a$, fatigue cracks propagate faster for the higher stress level at the same biaxiality. At a given stress level, fatigue cracks grow more rapidly with out-of-phase or negative biaxialities $(\lambda=-0.5$ and -1$)$ than in the uniaxial case, and slightly slower for in-phase cases or positive biaxialities $(\lambda=+0.5$ and +1.0$)$. These trends essentially differ from the results obtained at low levels of biaxial stress for the same specimen configuration [9], for which the effects of $\Delta \sigma$

(a) $\Delta \sigma_{1}=380 \mathrm{MPa}$

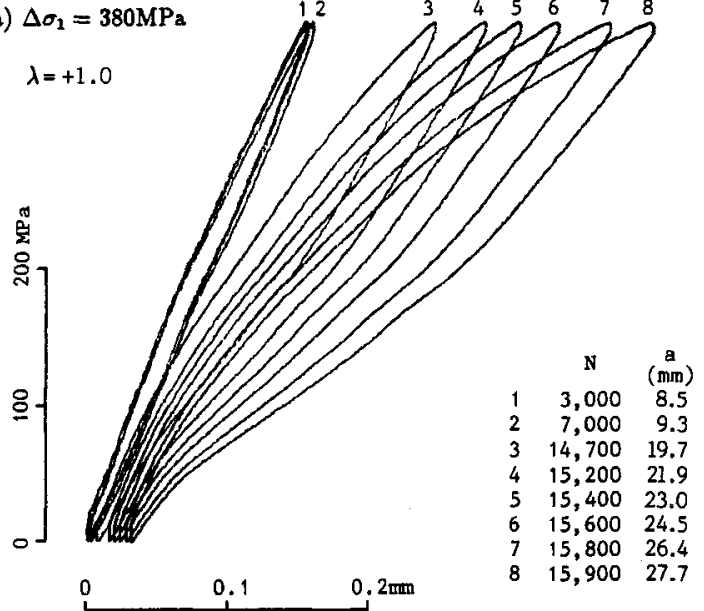

(b) $\Delta \sigma_{1}=560 \mathrm{MPa}$

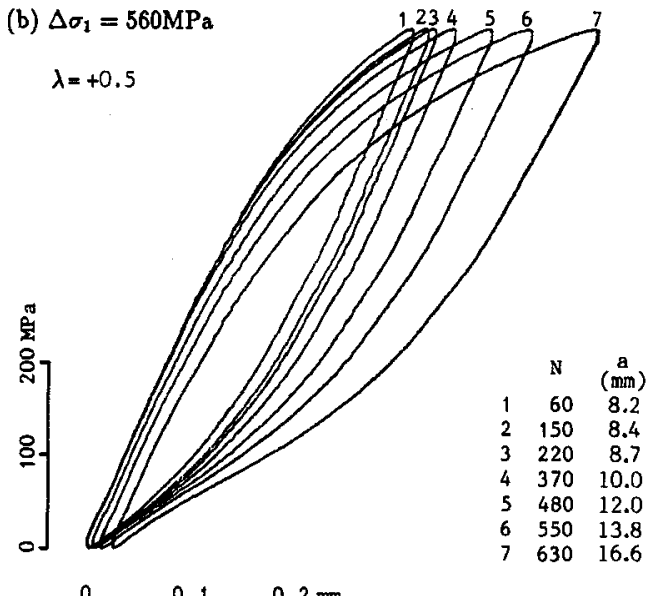

Fig. 6. Two examples of load-displacement hysteresis loops, for (a) $\Delta \sigma_{1}=380 \mathrm{MPa}$ with $\lambda=+1$ and (b) $\Delta \sigma_{1}=560 \mathrm{MPa}$ with $\lambda=+0.5$. 

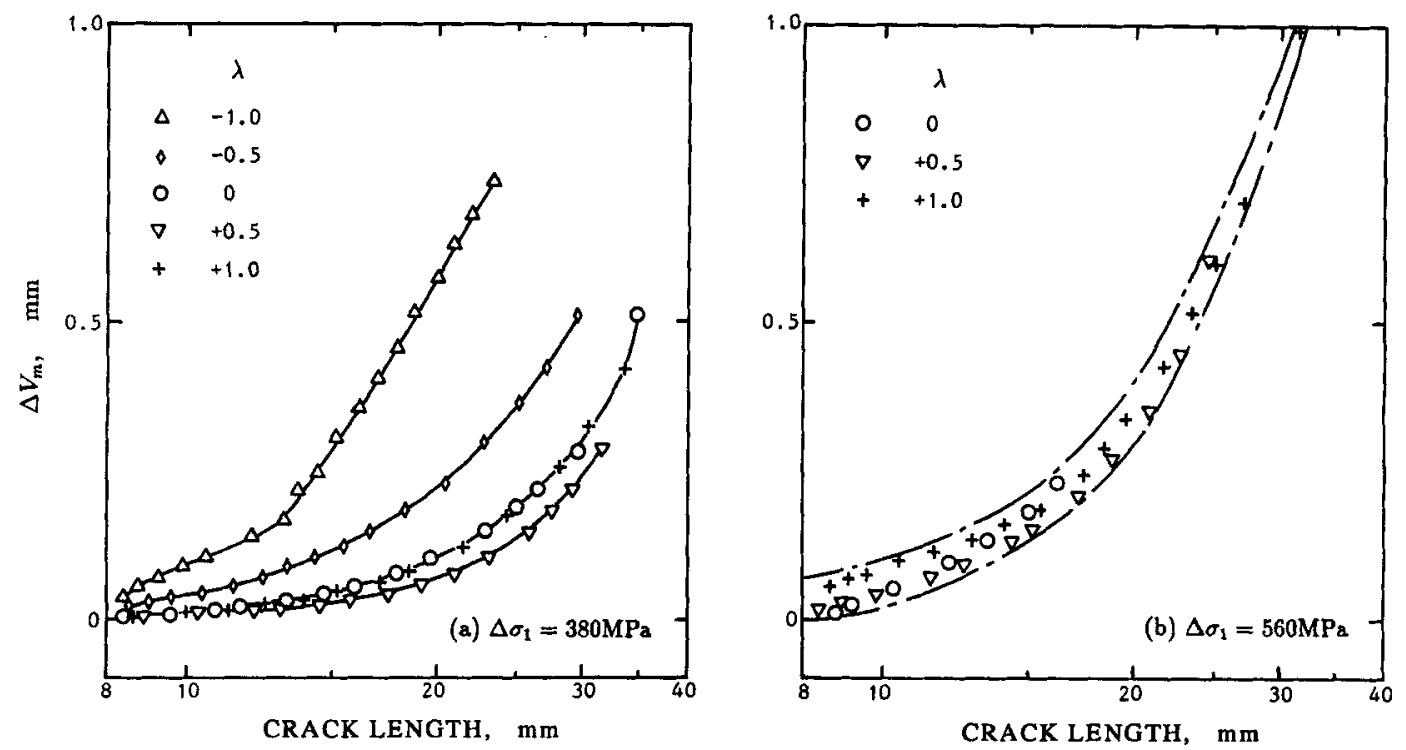

Fig. 7. Net changes of the maximum value of gauge length displacement for the $y$-axis as a function of crack length $a$, for (a) $\Delta \sigma_{1}=380 \mathrm{MPa}$ and (b) $\Delta \sigma_{1}=560 \mathrm{MPa}$.

and $\lambda$ on $\mathrm{d} a / \mathrm{d} N$ were well described by the stress intensity factor range. Thus the Paris law can be used to describe crack extension and predict fatigue life. However, in the present situation, the Paris law is inapplicable simply because the high stress leads to violation of the requirements postulated by LEFM, and because it does not include the plastic strain range.

There seems to be a paradox that, over a large range of fatigue crack growth rates, $\mathrm{d} a / \mathrm{d} N$ for $\lambda=+0.5$ is a little lower than that for $\lambda=+1.0$ at both stress levels. This arose when $a / b>1.50$ or $\Delta \sigma_{1} \sqrt{\pi a}(1+F)>70 \mathrm{MPa} \sqrt{\mathrm{m}}$ for $\Delta \sigma_{1}=380 \mathrm{MPa}$ [Figs 3(a) and 4(a)], and when $a / b>1.25$ or $\Delta \sigma_{1} \sqrt{\pi} a(1+F)>60 \mathrm{MPa} \sqrt{\mathrm{m}}$ for $\Delta \sigma_{1}=560 \mathrm{MPa}$ [Figs 3(b) and 4(b)]. This paradox may be interpreted in the following manner.

An interpretation of the influence of $\Delta \sigma$ and $\lambda$ on fatigue behaviour in this regime may be described in terms of the effective stress, $\Delta \sigma_{e}$, which is an appropriate representation of stresses in a biaxial state and can be expressed as (for a von Mises flow rule)

$$
\sigma_{\mathrm{e}}=\sigma_{1} \sqrt{1-\lambda+\lambda^{2}} \text {. }
$$

Obviously, the contribution of $\lambda$ to $\sigma_{\mathrm{e}}$ is in the form of $\sqrt{1-\lambda+\lambda^{2}}$ and their correlation is illustrated by Fig. 8. It is seen that, when $\lambda=+0.5$, the value of $\sqrt{1-\lambda+\lambda^{2}}$ reaches its minimum at 0.866 . Since the present tests were performed under high levels of biaxial stress, the fatigue crack behaviour is much more controlled by the plastic deformation in the vicinity of crack tip. Using the von Mises yielding criterion

$$
\sigma_{\mathrm{e}}=\sigma_{\mathrm{ys}}
$$

where $\sigma_{y s}$ is the yield stress under unaxial tension, one can see that the condition for plastic deformation is directly connected with both stress magnitude and biaxiality. On the other hand, according to the Prandtl-Reuss relations for plastic deformation and von Mises criterion, we may write

$$
\Delta \epsilon_{\mathrm{ep}}=\frac{2 \sqrt{1-\lambda+\lambda^{2}}}{2-\lambda} \Delta \epsilon_{\mathrm{lp}} .
$$




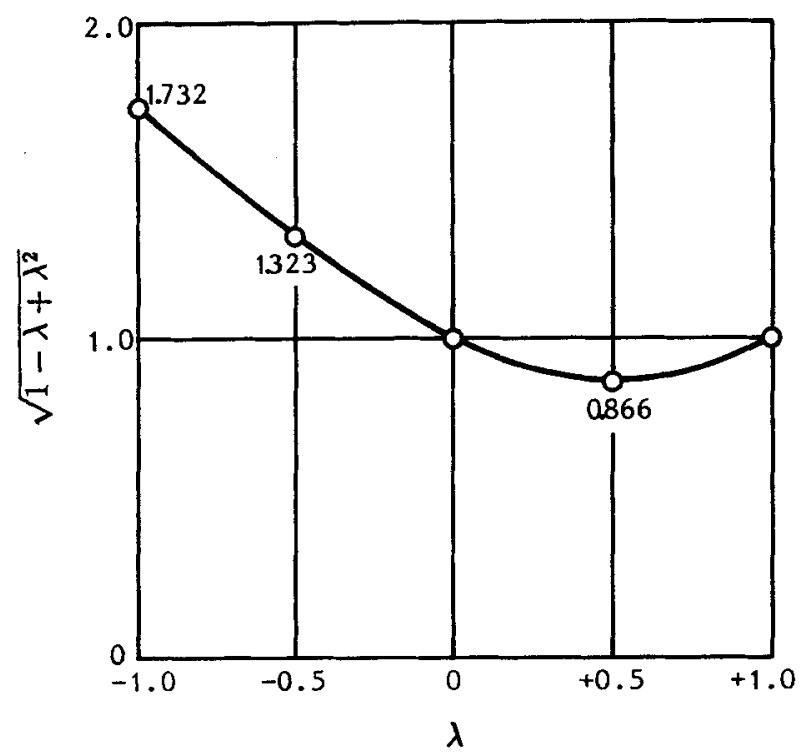

Fig. 8. Variation of $\sqrt{1-\lambda+\lambda^{2}}$ with $\lambda$.

Furthermore, one may assume that the effective stress, $\sigma_{\mathrm{e}}$, and the effective plastic strain, $\epsilon_{\mathrm{ep}}$, conform to a power law

$$
\sigma_{\mathrm{e}}=k^{\prime} \epsilon_{\mathrm{ep}}^{n^{\prime}}
$$

Our experimental results for the $\Delta \sigma_{1}$ versus $\Delta \epsilon_{1 \mathrm{p}}$ relations do agree with this assumption (Fig. 9) by giving the following linearly regressed formula

$$
\Delta \sigma_{\mathrm{e}}=1.80 \times 10^{3} \Delta \epsilon_{\mathrm{ep}}^{0.180}
$$

for which the range of $\Delta \sigma_{\mathrm{e}}$ is between 320 and $650 \mathrm{MPa}$. Equation (20) was obtained from 21 data points of the five biaxialities with the correlation coefficient $r=0.950$ and the standard deviation of $\log \left(\Delta \sigma_{\mathrm{e}}\right), \mathrm{SD}=0.0468$. Thus, the higher the value of $\sigma_{\mathrm{e}}$, the larger the resulting value $\epsilon_{\mathrm{ep}}$ for the notch-crack field, and vice versa. The agreement of experimental results with the von Mises flow rule also implies that the capacity for plastic deformation reaches the lowest point when $\lambda=+0.5$ at a given principal stress level, $\Delta \sigma_{1}$. The above inference not only demonstrates the general trend of fatigue crack velocity at different biaxialities, but also gives rise to an explanation for the lower crack rate at $\lambda=+0.5$ which is attributed to the smaller capacity for plastic deformation compared with other biaxialities.

As mentioned earlier, the Tomkins' model was created to assess crack growth in the low-cycle fatigue regime. Although modifications have been made to his original formula to allow for the present geometry under biaxial loadings, the new expression [equation (9)] is still lacking general applicability since the key factor $\Delta \epsilon_{1 \mathrm{p}}$ is not properly defined. If $\Delta \epsilon_{1 \mathrm{p}}$ is regarded as the uniform plastic strain away from the notch and tip, it is not readily measured. To overcome this problem, we first consider the mechanism of shear decohesion in the Tomkins' model, where the plastic strain range represents the shear strain applied to the crack tip plastic zone. Therefore $\Delta \epsilon_{1 p}$ should be replaced by the effective plastic strain, $\Delta \epsilon_{\mathrm{ep}}$, for the biaxial stress condition, as shown in equation (18), since this is the effective shear strain obtained due to plastic flow. Secondly, we assume that

$$
\Delta \epsilon_{l \mathrm{p}}=C\left(\frac{V_{\mathrm{m}}}{V_{0}}\right)
$$


where $V_{\mathrm{m}}$ is the maximum value of gauge length displacement during each fatigue cycle, $V_{0}$ the original gauge length and $C$ a proportional coefficient. This is proposed to allow for the fact that fatigue crack growth is associated not only with the far field uniform plastic strain but also with the deformation in the vicinity of the cracked notch. Consequently, we further develop equation (9) as

$$
\frac{\mathrm{d} a}{\mathrm{~d} N}=C \frac{\pi^{2}}{8} \cdot \frac{(1+F)^{2}}{(1+2 n)} \cdot \frac{2 \sqrt{1-\lambda+\lambda^{2}}}{2-\lambda}\left(\frac{\Delta \sigma_{1}}{2 \sigma_{\mathrm{u}}}\right)^{2}\left(\frac{V_{\mathrm{m}}}{V_{0}}\right) a
$$

or

$$
\frac{\mathrm{d} a}{\mathrm{~d} N}=C H\left(\lambda, V_{\mathrm{m}}, a\right)
$$

Substituting the present data into equation (22), we are able to show the results of $\mathrm{d} a / \mathrm{d} N$ as a function of $H$ for the five biaxialities at the two stress levels (Fig. 10), for $9.5 \leqslant a \leqslant 30 \mathrm{~mm}$. It is observed that the data resulting from $\lambda=-1,-0.5$ and 0 do present a good linear trend in a $\log -\log$ scale with a slope of unity, indicating a stable constant $C$ exists for these cases. The corresponding $C$ values are also shown in Fig. 10. Therefore, equation (23) can describe fatigue crack growth from circular notches under high levels of biaxial stress at $\lambda=-1.0,-0.5$ and 0 .

For the cases of positive biaxialities $(\lambda=+0.5$ and +1.0$)$, although linear trends between $\mathrm{d} a / \mathrm{d} N$ and $H$ still exist, the slopes of these lines are in excess of unity. In other words, equation (23) with a constant value of $C$ cannot represent these results. This may suggest that, for in-phase biaxial loading $(\lambda=+0.5$ and +1.0$)$, the fraction of plastic strain within the total displacement steadily

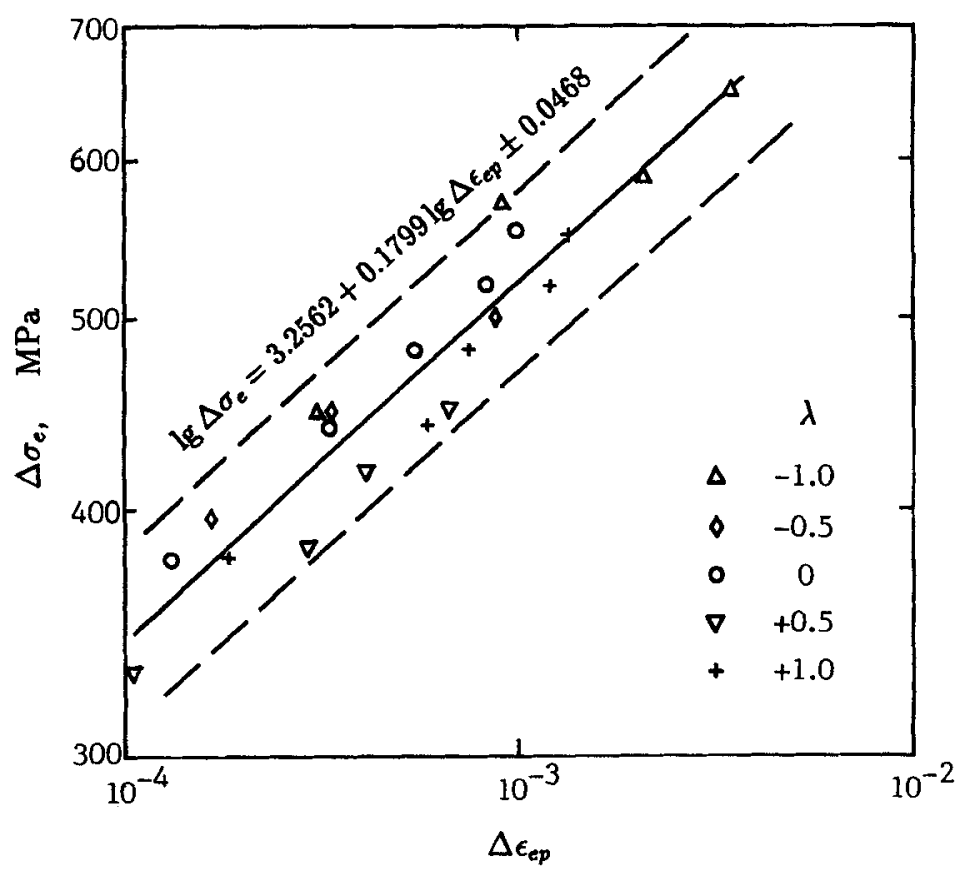

Fig. 9. Relationship between $\Delta \sigma_{\mathrm{e}}$ and $\Delta \epsilon_{\mathrm{ep}}$. 

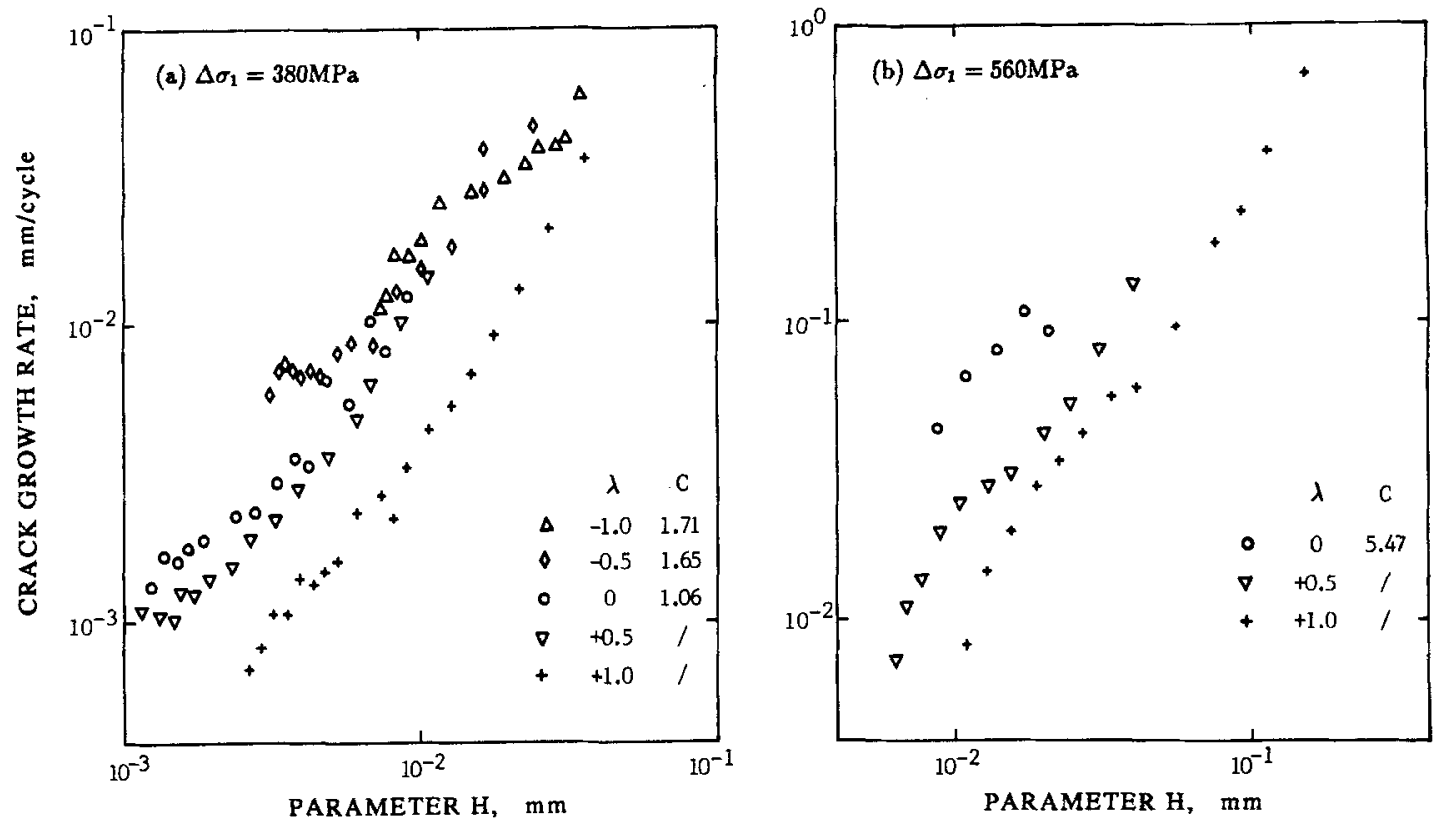

Fig. 10. Modified model describing crack growth rate as a function of $H$, for (a) $\Delta \sigma_{1}=380 \mathrm{MPa}$ and (b) $\Delta \sigma_{1}=560 \mathrm{MPa}$.

increases with the advance of crack length under a given cyclic stress range. On examining the tendency of these results, an empirical equation may be proposed

$$
\frac{\mathrm{d} a}{\mathrm{~d} N}=C^{\prime} H^{m}
$$

Consequently, fatigue crack propagation from circular notches under high levels of biaxial stress at positive biaxialities could be described by equation (24), where $C^{\prime}$ is a suitable function of stress biaxiality.

\section{CONCLUSIONS}

The following conclusions are drawn from the present investigation of fatigue crack growth from circular notches under high levels of biaxial stress.

(1) Fatigue crack growth rates are substantially affected by both the stress amplitude and the stress biaxiality. Crack growth is faster in the case of negative biaxialities and slightly slower for positive biaxialities.

(2) The highest fatigue crack growth rate occurs when $\lambda=-1.0$ and the lowest rate arises when $\lambda=+0.5$, which has been ascribed to the largest and the smallest plastic deformation capacity, respectively, possessed by the two biaxialities at a given principal stress level.

(3) The Tomkins' model is applicable to fatigue crack growth from circular notches under high levels of stress but further analysis is required on the effects of stress biaxiality. 


\section{REFERENCES}

1. P. C. Paris and F. Erdogan (1963) A critical analysis of crack propagation laws. Trans. Am. Soc. Mech. Engrs, Ser. D 85, 528-534.

2. R. C. Boettner, C. Laird and A. J. McEvily (1965) Crack nucleation and growth in high strain-low cycle fatigue. Trans. Metall. Soc. Am. Inst. Mining Metall. Petroleum Engrs 233, 379-387.

3. J. R. Haigh and R. P. Skelton (1978) A strain intensity approach to high temperature fatigue crack growth and failure. Mater. Sci. Engng 36, 133-137.

4. N. E. Dowling (1977) Crack growth during low cycle fatigue of smooth axial specimens. In Cyclic Stress-Strain and Plastic Deformation Aspects of Fatigue Crack Growth, ASTM STP 637, pp. 97-121. ASTM, Philadelphia, Pa.

5. M. S. Starkey and R. P. Skelton (1982) A comparison of the strain intensity and cyclic $J$ integral approaches to crack growth. Fatigue Engng Mater. Struct. 5, 329-341.

6. B. Tomkins (1984) High strain fatigue. In Sub-critical Crack Growth due to Fatigue, Stress Corrosion and Creep (Edited by L. H. Larsson), pp. 239-266. Elsevier Applied Science, London.

7. B. Tomkins (1968) Fatigue crack propagation-an analysis. Phil. Mag. 18, 1041-1066.

8. J. Wareing (1983) Mechanisms of high temperature fatigue and creep-fatigue failure in engineering materials. In Fatigue at High Temperature (Edited by R. P. Skelton), pp. 135-185. Applied Science, London.

9. S. T. Xiao and M. W. Brown (1987) Fagitue crack growth in notched AISI 316 stainless steel plates under biaxial loading. In Mechanical Behaviour of Materials (Edited by M. Yan, S. Zhang and Z. Zheng), Proceedings of ICM5, Beijing, June 1987, pp. 659-665. Pergamon Press, Oxford.

10. C. D. Hopper and K. J. Miller (1977) Fatigue crack propagation in biaxial stress fields. J. Strain Anal. $12,23-28$.

11. D. S. Dugdale (1960) Yielding of steel sheets containing slits. J. Mech. Phys. Solids 8, 100-104.

12. B. Tomkins (1975) The development of fatigue crack propagation models for engineering applications at elevated temperatures. J. Engng Mater. Technol. Trans. Am. Soc. Mech. Engrs Ser. H 97, 289-297.

13. Hong Youshi, K. J. Miller and M. W. Brown (1991) Complex stress functions and plastic zone sizes for notch cracks subjected to various loading conditions. Fatigue Fract. Engng Mater. Struct. 14, 237-258.

14. J. C. Newman (1971) An improved method of collocation for the stress analysis of cracked plates with various shaped boundaries. NASA TN D-6376.

15. S. T. Xiao, M. W. Brown and K. J. Miller (1985) Stress intensity factors for cracks in notched finite plates subjected to biaxial loading. Fatigue Fract. Engng Mater. Struct. 8, 349-372.

16. ASTM Standard E647-83 (1983) Constant load amplitude fatigue crack growth rates above $10^{-8} \mathrm{~m} /$ cycle. Appendix XI. 